\title{
On the calculation and use of non-zero momentum correlators in lattice simulations.
}

\author{
Claudio Rebbi* \\ Boston University \\ E-mail: rebbi@bu.edu
}

In lattice simulations one generally projects correlators over zero spatial momentum to calculate masses and related spectral data. The sum over space lattice points, however, discards information which may be useful especially in the calculation of disconnected diagrams. By using momentum conservation, the calculation of non-zero momentum components of disconnected diagrams and other quantities related to space convolutions can be done with little additional computational cost and may be useful in the analysis of disconnected correlators.

The 36th Annual International Symposium on Lattice Field Theory - LATTICE2018

22-28 July, 2018

Michigan State University, East Lansing, Michigan, USA.

${ }^{*}$ Speaker. 


\section{Introduction}

In lattice gauge theory simulations the calculation of correlators of particle whose source and sink have non-zero vacuum expectation value is challenging because of the need to include disconnected contributions. This happens, for example, in the calculation of glueball correlators and, most notably, in the calculation of the correlator of a flavor-singlet scalar particle. In this case the disconnected component involves a correlation of local operators:

$$
D(t)=\left(1 / N_{x} N_{t}\right) \sum_{\vec{x}, \vec{y}, t^{\prime}}\left\langle\phi\left(\vec{x}, t^{\prime}\right) \phi\left(\vec{y}, t^{\prime}+t\right)\right\rangle
$$

where $\phi\left(\vec{x}, t^{\prime}\right)$ is a closed fermion propagator, i.e. the trace of a fermion propagator originating and ending at $\vec{x}, t^{\prime}$. In Eq. 1.1, as is commonly done, one sums over the space positions $\vec{x}, \vec{y}$ of $\phi$, in order to project the correlator over zero momentum states. As a matter of fact a single sum, over $\vec{x}$ or $\vec{y}$, would in principle be sufficient to project over zero momentum, but the correlation of the individual terms in Eq. 1.1 is affected by large statistical fluctuations, which one reduces by summing over both $\vec{x}$ and $\vec{y}$. Still, for lattices of large size, calculating all the individual closed propagators in Eq. 1.1 over all the lattice configurations produced in the simulation would be too time consuming, and one resorts to suitable computational techniques, such as stochastic source methods, to accelerate the calculation. We will not need to be concerned here with these techniques. The only important fact for us is that, prior to the sums over $\vec{x}$ and $\vec{y}$, they provide a reasonably precise evaluation of the individual closed propagators at all points of the lattice.

For simplicity from now on we will refer to the disconnected component $D(t)$ of the correlator simply as the "disconnected correlator". Correspondingly, we will use the term "connected correlator" to indicate the connected component $C(t)$ of the correlators, where a fermion propagator from $\vec{x}, t^{\prime}$ to $\vec{y}, t^{\prime}+t$ is combined with a propagator from $\vec{y}, t^{\prime}+t$ to $\vec{x}, t^{\prime}$, with a suitable sum over spin and flavor indices. Also, we will leave normalization factors implicit.

The main point of this note is that, for large separation, $\phi\left(\vec{x}, t^{\prime}\right)$ and $\phi\left(\vec{y}, t^{\prime}+t\right)$ in Eq. 1.1 are essentially uncorrelated but still contribute to the noise. As, with the advance of computer power, simulations with lattices of increasingly larger size become possible, the problem grows more severe. E.g. with $N_{x}=256$ for $t=32$ the separation between $\phi\left(\vec{x}, t^{\prime}\right)$ and $\phi\left(\vec{y}, t^{\prime}+t\right)$ ranges from 32 to $\sqrt{32^{2}+3 \times 128^{2}}=224$ lattice spacings. We may then try to reduce the statistical noise by giving higher weight to the operators facing each other, and this will be our first consideration. The above weighting procedure, which involves a convolution, is most easily done in momentum space. As we shall see, a byproduct is that one can easily calculate the disconnected correlators for non vanishing momentum, and this will presented in a subsequent section.

\section{Gaussian weighted correlators}

We try to reduce the noise due to far away $\phi$ operators by correlating $\phi(\vec{x}, t)$ with a distribution of $\phi\left(\vec{y}, t^{\prime}\right)$ centered around $\vec{x}$ rather then the whole time slice at $t^{\prime}$. The most obvious distribution is a Gaussian one, although one could use different weight factors. Thus we define a "Gaussian weighted correlator" as

$$
D_{G}(t)=\sum_{\vec{x}, \vec{y}, t^{\prime}}\left\langle\phi\left(\vec{x}, t^{\prime}\right) G(\vec{y}) \phi\left(\vec{x}+\vec{y}, t^{\prime}+t\right)\right\rangle
$$



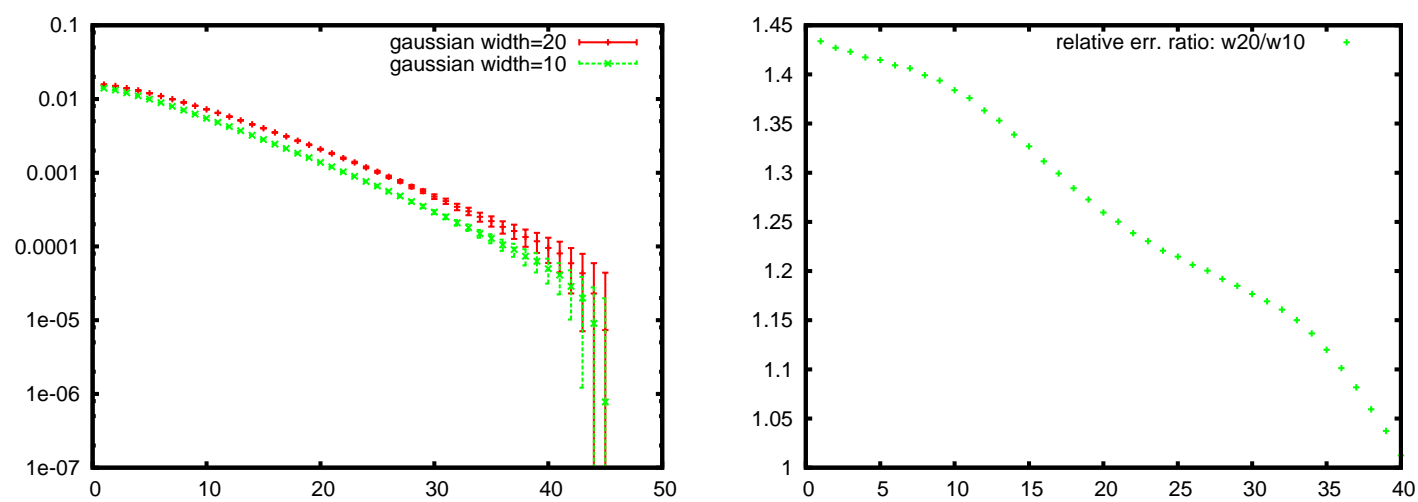

Figure 1: Left: The Gaussian weighted disconnected correlators discussed in the text for widths of 10 and 20 lattice spacings. Right: the ratio of relative errors for width 20 over the relative errors for width 10 .

where $G(\vec{y})$ is a Gaussian weight function falling off with distance. This may reduce the statistical noise at the expense of the introduction of non-zero momentum components.

Computing the above correlator in configuration space would be too demanding, it would involve the averaging over $N_{x}^{6}$ products of $\phi\left(\vec{x}, t^{\prime}\right)$ with $\phi\left(\vec{x}+\vec{y}, t^{\prime}+t\right)$, but the convolution in Eq. 2.1 can be much more readily computed in momentum space. We express $\phi(\vec{x}, t)$ and $G(\vec{x})$ in terms of their (space) Fourier transforms, which we denote by a tilde (we continue to neglect normalization factors in order to streamline the formulae)

$$
\begin{aligned}
\phi(\vec{x}, t) & =\sum_{\vec{p}} \tilde{\phi}(\vec{p}, t) e^{\imath \vec{p} \cdot \vec{x}} \\
G(\vec{x}) & =\sum_{\vec{p}} \tilde{G}(\vec{p}) e^{-l \vec{p} \cdot \vec{x}}
\end{aligned}
$$

and obtain

$$
D_{G}(t)=\sum_{\vec{x}, \vec{y}, \vec{p}, \vec{q}, \vec{r}, t^{\prime}} \tilde{G}(\vec{q})\left\langle\tilde{\phi}^{*}\left(\vec{p}, t^{\prime}\right) \tilde{\phi}\left(\vec{r}, t^{\prime}+t\right)\right\rangle e^{l[-\vec{q} \cdot \vec{y}-\vec{p} \cdot \vec{x}+\vec{r} \cdot(\vec{x}+\vec{y})]}
$$

(where we used the reality of $\phi(\vec{x}, t)$.) And now we make use of the crucial fact that correlators are diagonal in momentum. This property is not true configuration by configuration, but is true of the exact correlators, and will be better and better satisfied as one improves the statistics. So we assume it as true in Eq. 2.4. This introduces a delta function forcing $\vec{r}=\vec{p}$ and giving

$$
\begin{array}{r}
D_{G}(t)=\sum_{\vec{x}, \vec{y}, \vec{p}, \vec{q}, t^{\prime}} \tilde{G}(\vec{q})\left\langle\tilde{\phi}^{*}\left(\vec{p}, t^{\prime}\right) \tilde{\phi}\left(\vec{p}, t^{\prime}+t\right)\right\rangle e^{l[-\vec{q} \cdot \vec{y}+\vec{p} \cdot \vec{y})]} \\
=\sum_{\vec{p}, t^{\prime}} \tilde{G}(\vec{p})\left\langle\tilde{\phi}^{*}\left(\vec{p}, t^{\prime}\right) \tilde{\phi}\left(\vec{p}, t^{\prime}+t\right)\right\rangle
\end{array}
$$

From the computational point of view this equation leads to the following procedure. As one computes the closed fermion propagators $\phi(\vec{x}, t)$ configuration by configuration one should calculate right away their Fourier transforms $\tilde{\phi}(\vec{p}, t)$, or save the values of $\phi(\vec{x}, t)$ at all points $\vec{x}$ (rather than sum right away over $\vec{x}$ ) for a subsequent Fourier transform. The products of these Fourier transforms, averaged over all configurations, are then summed over $\vec{p}$ with the Gaussian weight factor $\tilde{G}\left(\tilde{)}\right.$ to obtain the Gaussian weighted correlators $D_{G}(t)$. For the full correlator of the state 
under consideration one should, of course, combine the disconnected correlator with the connected one with the appropriate coefficients, but the calculation of the Fourier transform of the connected correlator is straightforward.

We tested the above procedure on data for a scalar singlet correlator ( $\sigma$ particle) obtained by the LSD collaboration ${ }^{1}$ in the course of a study of the SU(3) gauge theory with eight dynamical fermions [1]. We refer to [1] for the detailed parameters of the simulation. The data we are showing have been obtained from averages over 2393 thermalized configurations, divided into three approximately equally numbered streams, on a $64 \times 128$ lattice with $\beta_{F}=4.8, \beta_{A} / \beta_{F}=-0.25$, and bare fermion mass $a m=0.00125$ in lattice units. A straightforward implementation of the Gaussian smearing would not be very revealing, because the fluctuations in $D(t)$ and $D_{G}(t)$ are dominated by the fluctuations of the vacuum expectation value of the disconnected correlators. However, the computational procedure outlined above permits to get rid very easily of the unwanted vacuum contribution. One only needs to exclude the value $\vec{p}=0$ from the sum in Eq. 2.5. In Figure 1 we show the disconnected $\sigma$ correlator weighted with Gaussians of widths $w=10$ and $w=20$ lattice units and with zero-momentum subtracted. With the large statistics the errors for $t \lesssim 30$ are not very visible in the graph at left, making it difficult to compare the relative accuracies of the two different Gaussian smearing, but in the graph at right we show the ratio of the relative errors in the data obtained with $w=20$ over those obtained with $w=10$. The results confirms that, as we would have expected, giving greater weight to the operators closer in space reduces the error due to statistical noise. As the separation in time increases the advantage gradually disappears, as could have also been expected, since for larger times the separation in time weighs more than the separation in space.

\section{Momentum dependent correlators}

A byproduct of the procedure described above is that it can also be used to easily calculate the individual momentum components of the disconnected correlators. Always under the assumption that momentum is conserved, which will be better and better satisfied as the statistics increases, the momentum components of the disconnected correlators are calculated by taking first the spatial Fourier transforms of the the closed fermion propagators as they are calculated configuration by configuration. Then the expectation value of the products of the Fourier transforms (FT) (more precisely of the FT and its conjugate) at definite time separation are averaged over all thermalized configurations. The disconnected correlators with at least one non-zero momentum component have no vacuum expectation value and including them in the overall analysis of the data may help improve the accuracy of the singlet scalar mass determination. Of course, the exponential behavior of the correlator (with disconnected and connected components combined) will provide a determination of the energy of the state rather than of its mass, so if the value of the lowest momentum is comparable or larger than the mass one wishes to determine, it will become more difficult to extract a reliable value for the mass from the correlators with non-vanishing momentum. Thus, as

\footnotetext{
${ }^{1}$ The Lattice Strong Dynamics collaboration: Xiao-Yong Jin, James Osborn (ANL); Richard Brower, Claudio Rebbi (BU); Pavlos Vranas (LLNL); Evan Weinberg (NVIDIA); Enrico Rinaldi (RBRC); David Schaich (U. Bern); Joseph Kiskis (UC Davis); Anna Hasenfratz, Ethan Neil (joint w/RBRC), Oliver Witzel (U. Colorado); Graham Kribs (U. Oregon); Thomas Appelquist, Kimmy Cushman, George Fleming, Andy Gasbarro (Yale).
} 


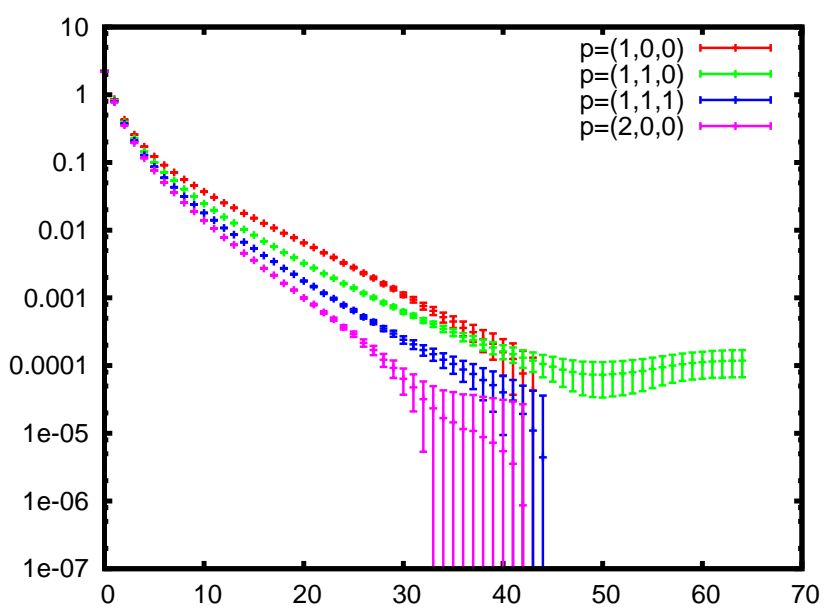

Figure 2: The full $\sigma$ correlators for the four lowest non-zero momenta from the simulations on a $64 \times 128$ lattice, $m=0.00125$, mentioned in the text.

in the case of the Gaussian weighted correlators, the use of non-zero momentum components will become more valuable as the size of the lattices that can be simulated will increase.

In Figure 2 we show the four lowest non-zero momentum components of the full correlator $2 * D(t)-C(t), C(t)$ being the connected correlator, of the $\sigma$ (scalar singlet) state in the analysis of the 2393 thermalized configurations, with lattice size $64 \times 128$ and bare fermion mass $a m=$ 0.00125 , referred to above.

Figure 3 shows single exponential fits to the data with uncorrelated errors for the $p=1,0,0$ component (left panel) and $p=1,1,0$ component (right panel - of course, as in Figure 2, all similar p components are averaged) over the range 20:30. From the corresponding coefficients of the exponential fall-offs, 0.1721 and 0.1678 for the lowest and next lowest momenta, one would derive bare mass values $a m=0.1413$ and $a m=0.0943$. The difference between the two values, the lowest one being in the middle of the error range for the $\sigma$ mass found in Ref. [1], the other one a little above the error range, points to the fact that substantial statistical fluctuations are still present in the data and validates the caution expressed by the large error quoted in Ref. [1]. The fits shown in Figure 3 are rather unsophisticated and should only be given illustrative value. The point which we would like to make is that it is rather easy to calculate the disconnected correlators (as well as the connected ones, of course) with non-zero momentum and that these can provide useful information supplementing the information obtained from zero momentum. The LSD collaboration is considering including non-zero momentum correlators in future analyses of the spectrum of masses.

\section{Conclusions}

Correlators with non-zero momentum in lattice simulations have been considered many times, so no originality is claimed for this. What may be new is the proposal to consider correlators 

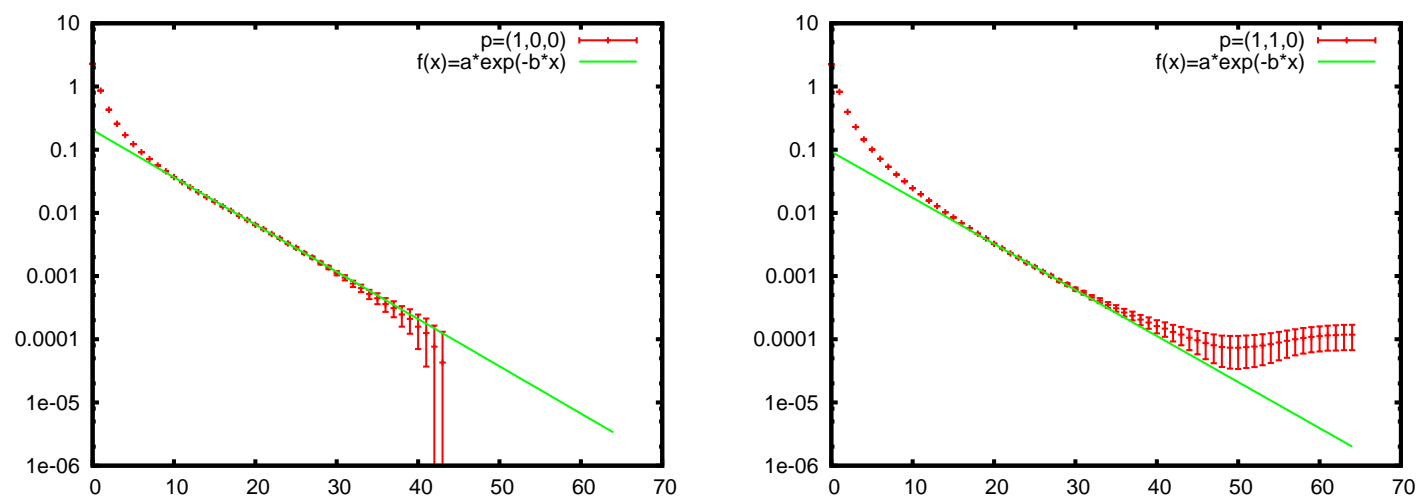

Figure 3: The correlators in Figure 2 for the two lowest momentum values with a single exponential fit with uncorrelated errors over the range $20: 30$.

weighted with a Gaussian distribution that favors the disconnected components at closer distances ${ }^{2}$. This noise reduction procedure is of limited or no value for lattices of small to moderate spatial extension, but may become useful as new, powerful computers permit the simulation of systems with very large spatial extent. Because of the inclusion of non-zero momentum, fits to the data become more elaborated, but can be done using a fitting function provided by a subroutine, rather than a combination of exponentials. Another point emphasized in this note is that the assumption of momentum conservation, which becomes better and better satisfied as the statistics of the simulation increases, allows a simplification in the calculation of the weighted correlators as well as of the momentum components of the disconnected correlators. Correlators with non-zero momentum avoid the need of a vacuum subtraction and their inclusion in a global fit may improve the overall accuracy in the extraction of spectroscopic data from the correlators.

\section{Acknowledgments}

I am grateful to my colleagues in the LSD collaboration for valuable discussions and, especially to Enrico Rinaldi and Evan Weinberg, for providing me with the raw data needed for my analysis of the correlators.

This work was supported by DOE grant DE-SC0015845.

\section{References}

[1] T. Appelquist, R. Brower, G. Fleming, A. Gasbarro, A. Hasenfratz, X.-Y. Jin, E. Neil, J. Osborn,

C. Rebbi, E. Rinaldi, D. Schaich, P. Vranas, E. Weinberg, and O. Witzel (Lattice Strong

Dynamics)(2018), arXiv:1807.08411 [hep-lat]

\footnotetext{
${ }^{2}$ I will appreciate being informed of any previous work that should be cited. If appropriate, I will include the citation in the article posted in the archives and, if feasible, in the published proceedings.
} 